\title{
Dynamics of a Family of Piecewise-Linear Area-Preserving Plane Maps I. Rational Rotation Numbers
}

\author{
Jeffrey C. Lagarias \\ Department of Mathematics \\ University of Michigan \\ Ann Arbor, MI 48109-1043 \\ email: lagarias@umich.edu \\ Eric Rains \\ Department of Mathematics \\ University of California-Davis \\ Davis, CA 95616-8633 \\ email: rains@math.ucdavis.edu
}

(August 2, 2006 version)

\begin{abstract}
This paper studies the behavior under iteration of the maps $T_{a b}(x, y)=\left(F_{a b}(x)-y, x\right)$ of the plane $\mathbb{R}^{2}$, in which $F_{a b}(x)=a x$ if $x \geq 0$ and $b x$ if $x<0$. The orbits under iteration correspond to solutions of the nonlinear difference equation $x_{n+2}=1 / 2(a-b)\left|x_{n+1}\right|+1 / 2(a+b) x_{n+1}-x_{n}$. This family of piecewise-linear maps has the parameter space $(a, b) \in \mathbb{R}^{2}$. These maps are area-preserving homeomorphisms of $\mathbb{R}^{2}$ that map rays from the origin into rays from the origin. The action on rays gives an auxiliary map $S_{a b}: S^{1} \rightarrow S^{1}$ of the circle, which has a well-defined rotation number. This paper characterizes the possible dynamics under iteration of $T_{a b}$ when the auxiliary map $S_{a b}$ has rational rotation number. It characterizes cases where the map $T_{a b}$ is a periodic map.
\end{abstract}

Keywords: area preserving map, iterated map, discrete Schrödinger operator AMS Subject Classification: Primary: 37E30 Secondary: 52C23, 82D30

\section{Introduction}

We study the behavior under iteration of the two parameter family of piecewise-linear homeomorphisms of $\mathbb{R}^{2}$ given by

$$
T_{a b}(x, y)= \begin{cases}(a x-y, x) & \text { if } \quad x \geq 0 \\ (b x-y, x) & \text { if } \quad x<0\end{cases}
$$

The parameter space is $(a, b) \in \mathbb{R}^{2}$. This map can be written

$$
T_{a b}(x, y)=\left[\begin{array}{cc}
F_{a b}(x) & -1 \\
1 & 0
\end{array}\right]\left[\begin{array}{l}
x \\
y
\end{array}\right],
$$


in which

$$
F_{a b}(x)= \begin{cases}a & \text { if } \quad x \geq 0 \\ b & \text { if } \quad x<0\end{cases}
$$

we view elements of $(x, y) \in \mathbb{R}^{2}$ as column vectors. The formula (1.2) shows that $T_{a b}(x, y)$ is a homeomorphism, since

$$
\begin{aligned}
T_{a b}^{-1}(x, y) & =\left[\begin{array}{cc}
F_{a b}(y) & -1 \\
1 & 0
\end{array}\right]^{-1}\left[\begin{array}{l}
x \\
y
\end{array}\right] \\
& =\left[\begin{array}{cc}
0 & 1 \\
-1 & F_{a b}(y)
\end{array}\right]\left[\begin{array}{l}
x \\
y
\end{array}\right] .
\end{aligned}
$$

It preserves the area form $d \omega=d x \wedge d y$, and it also maps rays from the origin into rays from the origin.

The maps $T_{a b}$ have some claim to being the simplest family of nonlinear area-preserving maps of the plane, which gives one reason to study their dynamics under iteration. A second reason to consider the dynamics of $T_{a b}$ (in terms of the parameters $(a, b)$ ), is that iteration of a fixed map $T_{a b}$ encodes the solutions of the second-order nonlinear recurrence

$$
x_{n+2}=\mu\left|x_{n+1}\right|+\nu x_{n+1}-x_{n}
$$

via

$$
T_{a b}\left(x_{n+1}, x_{n}\right)=\left(x_{n+2}, x_{n+1}\right)
$$

in which

$$
\mu=\frac{1}{2}(a-b), \quad \nu=\frac{1}{2}(a+b) .
$$

This recurrence can be interpreted as a solution to the one-dimensional nonlinear difference equation of Schrödinger type

$$
-x_{n+2}+2 x_{n+1}-x_{n}+V_{\mu}\left(x_{n+1}\right) x_{n+1}=E x_{n+1},
$$

in which the "potential" $V_{\mu}(x)$ is

$$
V_{\mu}(x):=\left\{\begin{array}{cl}
\mu & \text { if } \quad x \geq 0 \\
-\mu & \text { if } \quad x<0
\end{array}\right.
$$

and the energy value $E$ is

$$
E:=2-\nu
$$

(Strictly speaking, the potential is $x V_{\mu}(x)$.) Holding the potential $V_{\mu}$ fixed and letting the parameter $\nu$ vary amounts to studying the set of solutions for all real energy values $E=2-\nu$.

In this paper and its sequels we study the orbits of $T_{a b}$ for all parameter values, viewing the results as giving information about the set $\Omega_{S B}$ ("semi-bounded") of parameter values having at least one nonzero bounded orbit, as well as the smaller set $\Omega_{B}$ ("totally bounded") of parameter values having all orbits bounded. The set $\Omega_{S B}$ can be viewed as an analogue of the fractal "butterfly" structure of bound state energies appearing in a model of Hofstadter 12] for conduction of electrons in a metal with a stong magnetic field, as explained below. Our parameters $(\mu, 2-\nu)=\left(\frac{1}{2}(a-b), 2-\frac{1}{2}(a+b)\right)$ play the role of $(\alpha, E)$ in Hofstadter's model. 
In this paper we get information about the set $\Omega_{P}$ of purely periodic maps, and in part II we obtain information about the set $\Omega_{R}$ of maps topologically conjugate to a rotation of the plane, in which case the map has invariant circles. We have

$$
\Omega_{P} \subset \Omega_{R} \subseteq \Omega_{B} \subseteq \Omega_{S B},
$$

it is known that $\Omega_{R}=\Omega_{B}$, as discussed below. We defer a detailed study of $\Omega_{S B}$ to part III, where we show that $\Omega_{S B}$ is a closed set which has certain properties like those ascribed to the Hofstadter "butterfly".

The dynamics of $T_{a b}$ under iteration includes the induced dynamics on rays, which is described by a circle map $S_{a b}$ (defined in $\S 2$ ), as well as a stretching/shrinking motion within rays. As is usual for circle maps, there is a dichotomy in the dynamics between the cases of rational rotation number and irrational rotation number. In this paper the main result is to classify the possible dynamics in case of rational rotation number. In the process we characterize parameter values where the motion is purely periodic; this was done earlier by Beardon, Bullett and Rippon [2], as discussed below. Our interest in purely periodic maps $T_{a b}$ was stimulated by the observation of Morton Brown [6] in 1983 that the recurrence $x_{n+1}=\left|x_{n}\right|-x_{n-1}$ has all solutions periodic of period 9; this is the case $\mu=1, \nu=0$ of (1.5). At the end of the paper we establish a few facts about the irrational rotation number case, which is considered in more detail in part II. In $\S 2$ we summarize the main results, and then establish them in $\S 3-\S 5$.

There has been substantial earlier work on the dynamics of these maps. In 1986 M. Herman [11. Chap. VIII] studied the dynamics of maps in the Froeschlé group $\mathbb{F}$, which he defined to be ([11, p. 217]) the group of homeomorphisms of the plane generated by the elements of $S L(2, \mathbb{R})$, together with the piecewise linear maps of the form

$$
G_{a b}=\left[\begin{array}{cc}
1 & 0 \\
F_{a b}(x) & 1
\end{array}\right]
$$

for real $a, b$, viewed as acting on column vectors. This group was named after Froeschlé 9], who numerically computed (apparant) invariant circles for certain maps in $\mathbb{F}$. The maps $T_{a b}$ belong to $\mathbb{F}$, since

$$
\left[\begin{array}{cc}
F_{a b}(x) & -1 \\
1 & 0
\end{array}\right]=\left[\begin{array}{cc}
-F_{-a,-b}(x) & -1 \\
1 & 0
\end{array}\right]=\left[\begin{array}{cc}
0 & -1 \\
1 & 0
\end{array}\right]\left[\begin{array}{cc}
1 & 0 \\
F_{-a,-b}(x) & 1
\end{array}\right] .
$$

Herman was in large part concerned with proving the existence of invariant circles for a large set of such maps, and we comment further on his work in part II. However he also conjectured the existence of maps in the Froeschlé group having dense orbits in the plane ([11, p. 221]). It remains an open question whether any maps $T_{a b}$ have this property.

In 1995 Beardon, Bullett and Rippon [2] studied periodic orbits of the maps (1.5). They also studied the set of parameter values $\Omega_{B}$ for which all orbits are bounded, determined restrictions on the parameters in this set, and observed that the equality $\Omega_{R}=\Omega_{B}$ follows from results of Herman [11, VIII.2.4]. Our methods and results in part I overlap considerably with theirs; more remarks on this are made in $\S 2$. The results and examples given here on periodic orbits were first obtained in their paper. We include our own proofs of these results for the reader's convenience, as our notation differs from that of [2], and because properties of certain families of periodic maps are needed in later proofs in parts II and III. Our main result here settles a question raised in their paper. 
We conclude this introduction with two remarks. First, we note that another extensively studied two-parameter family of plane maps which are piecewise-affine with two pieces are the Lozi maps, introduced by Lozi [17]. These maps have the form

$$
L(x, y)=(1-a|x|+y, b x),
$$

where $a, b$ are real and $b<0$. These maps are homeomorphisms of $\mathbb{R}^{2}$, but do not preserve area (except for $b=-1$ ), nor do they take rays through the origin to rays through the origin. Lozi maps are known to exhibit a wide range of chaotic behavior, including strange attractors, see Misiurewicz [18] and Collet and Levy [7. The maps we consider in this paper are much simpler than the Lozi maps, but our results show they have nontrivial behavior under iteration.

Second, we note that the difference equation (1.7) may be compared with two much-studied classes of operators.

(i) The nonlinear Schrödinger operator with a general (real-valued) potential $V$ takes the form

$$
-\Delta u+V(|u|) u=i \frac{\partial}{\partial t} u
$$

where the function $u(t, x)$ is complex-valued. For stationary phase solutions $u(t, x)=e^{i E t} z(x)$ the phase component factors out and leads to

$$
-\Delta z+V(|z|) z=-E z .
$$

The discretized version of this equation involves the discrete nonlinear Schrödinger operator

$$
\Phi_{V}(z)_{n}=-z_{n+2}+2 z_{n+1}-z_{n}-V\left(\left|z_{n+1}\right|\right) z_{n+1}
$$

in the eigenvalue equation

$$
-z_{n+2}+2 z_{n+1}-z_{n}-V\left(\left|z_{n+1}\right|\right) z_{n+1}=E z_{n+1},
$$

for $z_{n} \in \mathbb{C}$, see [26]. The special case of solutions to this operator with constant phase $\mathbf{z}_{n}=e^{1 \phi} x_{n}$ (with $0 \leq \phi<\pi, x_{n}$ real), reduces to the general form (1.7) with a symmetric potential $V(x)=V(-x)$ (by cancelling out all phases). The recurrence (1.7) that we consider has a similar form to (1.10), with the difference that the potential $V(x)$ is antisymmetric, i.e. $V(x)=-V(-x)$.

(ii) Discrete linear Schrödinger operators on the line have been extensively studied, as simple models for conduction/insulation transitions of elections in metals, and more recently in quasicrystals, see [3], 8], 12], 14], 23], 24], 25]. The linear difference operator

$$
-x_{n+2}+2 x_{n+1}-x_{n}+V(n+1) x_{n+1}=E x_{n+1},
$$

with a potential $V(n)$ depending only on position is often called the "tight-binding" approximation to the Schrödinger operator on the line. Here one is interested in characterizing the values of $E$ which have extended states these are bounded orbits, that is, real-valued orbits in $l_{\infty}(\mathbb{Z})$. (Extended states are required in the physics literature to not belong to $l_{2}$ but we do not impose this condition; states belonging to $l_{2}$ are called localized states.) We define the $l_{\infty}$-spectrum for a fixed potential $V$ to be

$$
\operatorname{Spec}_{\infty}[V]:=\{E: \text { The discrete Schrödinger equation (1.11) has a bounded orbit }\} .
$$


In 1976 Hofstadter [12] computed numerically the $l_{\infty}$-spectrum $\Sigma_{\lambda, \alpha}$ for the discrete Schrödinger operator with a quasiperiodic potential $V(n)=\lambda \cos (2 \pi \alpha n)$, holding $\lambda=2$ fixed, and letting $\alpha$ vary. (This equation models allowable conduction energies of an electron moving in a twodimensional cubic crystal with a strong magnetic field applied perpendicularly.) He observed that the spectrum formed a two dimensional picture in the $(\alpha, E)$ plane resembling a fractal "butterfly". He gave a a conjectural explanation for the fractal structure using a kind of renormalization. For fixed irrational $\alpha$ the $l_{\infty}$-spectrum appeared to be a Cantor set of measure zero. Hofstadter's model has been extensively studied, with one goal being to give a rigorous justification of this "butterfly" structure; see Bellissard [4] and Sjöstrand 22. This spectrum for fixed parameters $(\lambda, \alpha)$ has a "band" structure when $\alpha$ is rational. A slightly more general problem almost Mathieu equation, which considers the potential $V(n)=2 \lambda \cos (2 \pi((n \alpha+\theta))$ where $(\lambda, \alpha, \theta)$ are parameters. Much recent progress has been made on the $l^{2}$-spectrum of this equation as a function of the parameters, see Jitomirskaya [13 and Puig [21]. A solution to long-standing problem of Cantor set spectrum when $\alpha$ is irrational has apparantly been achieved by Avila and Jitomirskaya [1]. For general results on discrete Schrödinger operators see Bougerol and Lacroix [5] and Pastur and Figotin [20].

Notation. We write $\mathbf{v}=\left(\mathbf{v}_{x}, \mathbf{v}_{y}\right) \in \mathbb{R}^{2}$, to be viewed as a column vector. An interval $\left[\mathbf{v}_{1}, \mathbf{v}_{2}\right)$ of the unit circle, or corresponding sector $\mathbb{R}^{+}\left[\mathbf{v}_{1}, \mathbf{v}_{2}\right)$ of the plane $\mathbb{R}^{2}$, is the one specified by going counterclockwise from $\mathbf{v}_{1}$ to $\mathbf{v}_{2}$.

Acknowledgments. We did most of the work on this paper while employed at AT\&T LabsResearch, whom we thank for support; most of the results in parts I and II were obtained in the summer of 1993. We thank T. Spencer for helpful comments on the relation of (1.7) to nonlinear Schrödinger operators, and M. Kontsevich for bringing the work of Bedford, Bullett and Rippon [2] to our attention.

\section{Summary of Results}

The parameter space of the map can be taken to be either $(a, b)$ or $(\mu, \nu)$, as these are equivalent by

$$
\mu=\frac{1}{2}(a-b), \quad \nu=\frac{1}{2}(a+b) .
$$

Both coordinate systems have their advantages, and we write the map (1.1) as $T_{a b}, T_{\mu \nu}$ accordingly. It is convenient to represent the action of $T_{a b}$, acting on column vectors $\mathbf{v}_{n}=\left(x_{n+1}, x_{n}\right)$ as

$$
T_{n}\left(\mathbf{v}_{0}\right)=\left[\begin{array}{c}
x_{n+1} \\
x_{n}
\end{array}\right]=M_{n}\left(\mathbf{v}_{0}\right)\left[\begin{array}{l}
x_{1} \\
x_{0}
\end{array}\right]
$$

in which

$$
M_{n}\left(\mathbf{v}_{0}\right)=\prod_{i=1}^{n}\left[\begin{array}{cc}
F_{a b}\left(x_{i}\right) & -1 \\
1 & 0
\end{array}\right]:=\left[\begin{array}{cc}
F_{a b}\left(x_{n}\right) & -1 \\
1 & 0
\end{array}\right] \cdots\left[\begin{array}{cc}
F_{a b}\left(x_{2}\right) & -1 \\
1 & 0
\end{array}\right]\left[\begin{array}{cc}
F_{a b}\left(x_{1}\right) & -1 \\
1 & 0
\end{array}\right] .
$$

Conjugation by the involution $J_{0}:(x, y) \rightarrow(-x,-y)$ gives

$$
T_{b a}(x, y)=J_{0}^{-1} \circ T_{a b} \circ J_{0} .
$$


Thus, in studying dynamics, without loss of generality we can restrict to the closed half-space $\{(a, b): a \geq b\}$ of the $(a, b)$ parameter space. This corresponds to the region $\{(\mu, \nu): \mu \geq 0\}$ of the $(\mu . \nu)$ parameter space, with $T_{\mu \nu}$ conjugate to $T_{-\mu, \nu}$.

Conjugation by the involution $R:(x, y) \rightarrow(y, x)$ takes $T_{a b}$ to its inverse map $T_{a b}^{-1}$, i.e.

$$
T_{a b}^{-1}(x, y)=R^{-1} \circ T_{a b} \circ R
$$

see Theorem 3.4. Thus, the dynamics of running the iteration backwards is essentially the same as running it forwards.

The map $T_{a b}$ is homogeneous, so sends rays $[\mathbf{v}]:=\{\lambda \mathbf{v}: \lambda \geq 0\}$ to rays $T_{a b}([\mathbf{v}])$. Therefore we obtain a well-defined circle map $S_{a b}: S^{1} \rightarrow S^{1}$, for $0 \leq \theta \leq 2 \pi$, given by

$$
S_{a b}\left(e^{i \theta}\right):=\frac{T_{a b}\left(e^{i \theta}\right)}{\left\|T_{a b}\left(e^{i \theta}\right)\right\|}, \quad 0 \leq \theta \leq 2 \pi .
$$

where $\left\|T_{a b}\left(e^{i \theta}\right)\right\|$ is the Euclidean norm on $\mathbb{R}^{2}$, and we identity $e^{i \theta}=x+y i \in \mathbb{C}$ with $(x, y) \in \mathbb{R}^{2}$. By abuse of language we shall also sometimes treat the circle map as $S_{a b}(\theta)$ having domain $\mathbb{R} / 2 \pi \mathbb{Z}$. Understanding the dynamics of the map $T_{a b}$ subdivides into two problems: study of the dynamics of the circle map $S_{a b}$, and study of the motion of points inside the individual rays.

In $\S 3$ we study the circle map $S_{a b}$. It has a well-defined rotation number $r\left(S_{a b}\right)$, and we determine the allowed range of the rotation number, as follows.

Theorem 2.1 For fixed real $a$, and $-\infty<b<\infty$, the rotation number $r\left(S_{a b}\right)$ is continuous and nonincreasing in $b$, and completely fills out the following intervals.

(i) For $a<0$,

$$
r\left(S_{a b}\right) \in\left[0, \frac{1}{2}\right]
$$

(ii) Let $0 \leq a<2$. Then for each integer $n \geq 2$, on the interval $2 \cos \frac{\pi}{n} \leq a<2 \cos \frac{\pi}{n+1}$,

$$
r\left(S_{a b}\right) \in\left[0, \frac{1}{n+1}\right] .
$$

(iii) For $a \geq 2$ one has

$$
r\left(S_{a b}\right)=0
$$

We next determine the range of the rotation number in $(\mu, \nu)$-space, for fixed $\mu$.

Theorem 2.2 For fixed real $\mu$ and $-\infty \leq \nu<\infty$ the values of $r\left(S_{\mu \nu}\right)$ are continuous and nonincreasing in $\nu$ and completely fill out the closed interval

$$
r\left(S_{\mu \nu}\right) \in\left[0, \frac{1}{2}\right]
$$

We conclude $\S 3$ with examples of specific parameter sets where the rotation number can be determined exactly. These include one-parameter families, and cases where the map $T_{a b}$ is purely periodic. These examples were all given in Bedford, Bullett and Rippon [2].

In $\S 4$ we study maps $T_{a b}$ for which $r\left(S_{a b}\right)$ is rational. The maps $S_{a b}$ exhibit a "modelocking" behavior so that there are open sets in the parameter space for which $r\left(S_{a b}\right)$ takes a fixed rational value (for certain rationals), as in Theorem [2.1(iii) above. We first characterize those values of $(a, b)$ where $T_{a b}$ is periodic. 
Theorem $2.3 T_{a b}$ is of finite order if and only if the orbit of $(0,1)$ is periodic.

This result was obtained in Beardon, Bullett and Rippon [2, Theorem 3.1(i)]. This result implies that the parameter values at which $T_{a b}$ is a periodic map fall in a countable number of one-parameter families, plus a countable number of isolated values, described by the period and possible symbolic dynamics of the orbits, as explained in $\S 4$.

We then prove the main result of this paper, which characterizes the dynamics in all cases of rational rotation number, as follows.

Theorem 2.4 If the rotation number $r\left(S_{a b}\right)$ is rational, then $S_{a b}$ has a periodic orbit, and one of the following three possibilities occurs.

(i) $S_{a b}$ has exactly one periodic orbit. Then $T_{a b}$ has exactly one periodic orbit (up to scaling) and all other orbits diverge in modulus to $+\infty$ as $n \rightarrow \pm \infty$.

(ii) $S_{a b}$ has exactly two periodic orbits. Then $T_{a b}$ has no periodic orbits. All orbits of $T_{a b}$ diverge in modulus to $+\infty$ as $n \rightarrow \pm \infty$, with the exception of orbits lying over the two periodic orbits of $S_{a b}$. These exceptional orbits have modulus diverging to $+\infty$ in one direction and to 0 in the other direction, with forward divergence for one, and backward divergence for the other.

(iii) $S_{a b}$ has at least three periodic orbits. Then $T_{a b}$ is of finite order, i.e. $T_{a b}^{(k)}=I$ for some $k \geq 1$, and all its orbits are periodic.

This result answers a question raised in Beardon, Bullett and Rippon [2, p. 671]: Can there be a nonperiodic $T_{a b}$ having two disjoint orbits of rays on each of which $T_{a b}^{q}=I$ ? This corresponds to case (ii) above, so the answer is: no.

In $\S 5$ we begin the study of maps $T_{a b}$ for which the rotation number $r\left(S_{a b}\right)$ is irrational. We show that the existence of an orbit with elements of modulus bounded away from 0 and $\infty$ implies that there exists an invariant circle (Theorem [5.2). Then we prove that an invariant circle is necessarily preserved under the reflection symmetry $R(x, y)=(y, x)$ (Theorem 5.3). Part II makes a further study of maps with irrational rotation number and invariant circles. We note that in the irrational rotation number case a result of Herman [11. VIII.2.4] implies a dichotomy: either $T_{a b}$ is topologically conjugate to a rotation of the plane, or else it has a dense orbit. However in part III we show in the latter case that it also possesses a bounded orbit.

As remarked in the introduction, our methods and results overlap with those used in Beardon, Bullett and Rippon [2. Their paper contains additional results about periodic maps beyond those given here. To compare results, their parameters $(\lambda, \mu)$ correspong to our $(\mu, \nu)$; they lift the difference equation (1.5) to a map of the plane exchanging $x$ and $y$ coordinates from our map, so their rotation numbers are negative while ours are positive; their parameter sets $\Lambda_{P}, \Lambda_{R}, \Lambda_{P}$ correspond to our parameter sets $\Omega_{P}, \Omega_{R}, \Omega_{B}$, respectively. Theorem 2.4 is able to advance beyond their results using the uniqueness assertion in Lemma 4.1

\section{Associated Circle Map}

We study the circle map $S_{\mu \nu}(\theta)$ in the $(\mu, \nu)$-coordinates.

Theorem 3.1 Each $S_{\mu \nu}(\theta)$ is an orientation-preserving homeomorphism from $S^{1}$ to itself. The derivative $\frac{d}{d \theta}\left(S_{\mu \nu}(\theta)\right)$ is continuous and of bounded variation. 
Proof. Coordinatizing $S^{1}$ as $(\cos (\theta), \sin (\theta))$, we have

$$
S_{\mu \nu}(\theta)=\cot ^{-1}\left(\frac{\nu \cos (\theta)+\mu|\cos (\theta)|-\sin (\theta)}{\cos (\theta)}\right)+\pi[\cos (\theta)<0] .
$$

This is clearly continuous, mod $2 \pi$; the only cause for concern is when $\cos (\theta)=0$, where it can be easily verified that the pieces match up. Similarly, if we look at the derivative of $S_{\mu \nu}$, we get:

$$
\frac{d}{d \theta} S_{\mu \nu}(\theta)=\frac{1}{(\nu \pm \mu)^{2} \cos ^{2}(\theta)-2(\nu \pm \mu) \cos (\theta) \sin (\theta)+1},
$$

with + when $\cos (\theta)>0$ and - when $\cos (\theta)<0$. This is piecewise continuous, and again, the pieces match up. Finally, the second derivative of $S_{\mu \nu}$ exists except when $\cos (\theta)=0$, and is bounded. We have

$$
\frac{d^{2}}{d \theta^{2}} S_{\mu \nu}(\theta)=\frac{(\nu \pm \mu)^{2} \sin (2 \theta)+2(\nu \pm \mu) \cos (2 \theta)}{\left((\nu \pm \mu)^{2} \cos ^{2}(\theta)-2(\nu \pm \mu) \cos (\theta) \sin (\theta)+1\right)^{2}},
$$

and the numerator is bounded by

$$
\left|(\nu \pm \mu)^{2} \sin (2 \theta)+2(\nu \pm \mu) \cos (2 \theta)\right| \leq(|\nu|+|\mu|) \sqrt{(|\nu|+|\mu|)^{2}+4}
$$

and the denominator by

$\left|(\nu \pm \mu)^{2} \cos ^{2}(\theta)-2(\nu \pm \mu) \cos (\theta) \sin (\theta)+1\right|^{-1} \leq 1+\frac{1}{2}\left((|\nu|+|\mu|)^{2}+(|\nu|+|\mu|) \sqrt{(|\nu|+|\mu|)^{2}+4}\right)$.

Thus, we can conclude that $\frac{d}{d \theta} S_{\mu \nu}(\theta)$ is continuous and of bounded variation. Finally, $S_{\mu \nu}$ is orientation-preserving, since $\frac{d}{d \theta} S_{\mu \nu}(\theta)$ is always positive, and is a homeomorphism, since $S_{\mu \nu}^{-1}$ obtained by conjugating $S_{\mu \nu}$ by reflection through the line $x=y$.

The dynamics of iteration of maps on the circle is well understood. A basic result (19. p.33]) is that every orientation-preserving homeomorphism $S: S^{1} \rightarrow S^{1}$ has a well-defined rotation number, defined by

$$
r(S):=\lim _{n \rightarrow \infty} \frac{\tilde{S}^{(n)}(x)-x}{n},
$$

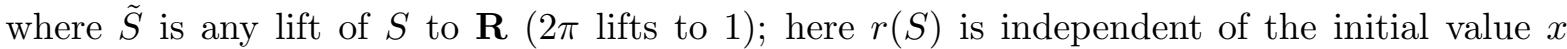
$(\bmod 1)$ and of the choice of lift. (Positive rotation number corresponds to counterclockwise rotation.) In particular, if $S$ has a periodic point, then $r(S)$ is rational (and conversely, as well).

This section gives general results on the rotation number $r\left(S_{a b}\right)$; the following two sections cover the case $r\left(S_{a b}\right)$ rational and $r\left(S_{a b}\right)$ irrational, respectively.

Theorem 3.2 (i) For fixed $b$, the rotation number $r\left(S_{a b}\right)$ is nonincreasing in a, and for fixed $a$ it is nonincreasing in $b$.

(ii) For fixed $\mu$, the rotation number $r\left(S_{\mu \nu}\right)$ is nonincreasing in $\nu$. 
Proof. (i). It suffices to show that $r\left(S_{a b}\right)$ is nonincreasing in $a$, the result for $b$ follows from the relation $r\left(S_{a b}\right)=r\left(S_{b a}\right)$.

Now, consider the behavior of $S_{a b}(\theta)$ as $a$ increases. If we can show that it moves clockwise as $a$ increases, for every $\theta$, then we are done, by inspection of equation (3.1). To show that $S_{a b}(\theta)$ moves clockwise with $a$, we need only show that

$$
L=T_{a b}(\mathbf{v})_{x} \frac{\partial}{\partial a} T_{a b}(\mathbf{v})_{y}-T_{a b}(\mathbf{v})_{y} \frac{\partial}{\partial a} T_{a b}(\mathbf{v})_{x} \leq 0
$$

for all $\mathbf{v} \in \mathbb{R}^{2}$. For $\mathbf{v}_{x}<0$, this is clearly 0 , since $T_{a b}(\mathbf{v})$ is independent of $a$ in that case. For $\mathbf{v}_{x} \geq 0, T_{a b}(\mathbf{v})=\left(a \mathbf{v}_{x}-\mathbf{v}_{y}, \mathbf{v}_{y}\right)$; a simple calculation gives

$$
L=-\mathbf{v}_{x}^{2} \leq 0
$$

so (i) is proved.

(ii). As $\nu$ increases, both $a$ and $b$ increase, so the result essentially follows from (i). More precisely, for fixed $\theta$ the point $S_{\mu \nu}(\theta)$ moves clockwise as $\nu$ increases.

It a natural quesion to ask how the rotation number varies when $\nu$ is held fixed, and $\mu$ varies. Numerical evidence indicates that it is nonincreasing for $\mu>0$, hence nondecreasing for $\mu<0$ (recall that $r\left(S_{\mu \nu}\right)=r\left(S_{-\mu, \nu}\right)$ since $T_{\mu \nu}$ is topologically conjugate to $T_{-\mu, \nu}$ ). This was observed by Bedford, Bullett and Rippon [2], who proved this holds for $\nu=0$. One can show that the rotation number is nonincreasing for $\mu>|\nu|$, using an extension of the approach used in Theorem 3.2(i). One checks for fixed $\theta$ that the fourth iterate $S_{\mu \nu}^{(4)}(\theta)$ moves clockwise as $\mu$ increases, with $\nu$ held fixed; there are 16 possible cases to consider, depending on the signs of the iterates. However new ideas seemed needed to determine how the rotation number behaves in the region $0<\mu<|\nu|$.

The fact that the rotation number is monotonic in $b$ allows us to give fairly strong bounds on the rotation number, for a given $a$, as follows.

Theorem 3.3 For each fixed $a$ and $-\infty \leq b \leq \infty$ the values of $r\left(S_{a b}\right)$ completely fill out the following intervals, including their endpoints.

(i) For $a<0$, the rotation number satisfies

$$
r\left(S_{a b}\right) \in\left[0, \frac{1}{2}\right]
$$

(ii) Let $0 \leq a \leq 2$. For each $n \geq 2$ on the interval $2 \cos \frac{\pi}{n} \leq a<2 \cos \frac{\pi}{n+1}$, the rotation number satisfies

$$
r\left(S_{a b}\right) \in\left[0, \frac{1}{n+1}\right] .
$$

(iii) For $a \geq 2$, the rotation number satisfies

$$
r\left(S_{a b}\right)=0
$$


Proof. We first consider the lower bounds in (i)-(iii). Now $r\left(S_{a b}\right)$ is nonincreasing in $b$, and $S_{a b}$ has a fixed point whenever $b \geq 2$; the existence of a fixed point implies $r\left(S_{a b}\right)=0$. This establishes the lower bound and shows it is attained for all real $a$.

For the upper bound in case (iii), if $a \geq 2$ then $r\left(S_{a b}\right)=0$ since $r\left(S_{a b}\right)=r\left(S_{b a}\right)$ by the conjugacy (2.4), and $r\left(S_{b a}\right)=0$.

For the upper bound in the other cases, these will follow if we show they are attained for all sufficiently large negative $b$. Suppose first that $-2 \leq a \leq 2$, and define $\theta$ so that $a=2 \cos \theta$, $0 \leq \theta \leq \pi$. Let $x_{i}(\mathbf{v})=\left(T_{a b}^{(i)} \mathbf{v}\right)_{x}$. Then we claim that, for $b$ sufficiently small, there is a periodic point $\mathbf{v}$ of $S_{a b}$ such that $\mathbf{v}_{x}<0, x_{i}(\mathbf{v})>0,1 \leq i \leq n$, and $S_{a b}^{(n+1)}(\mathbf{v})=(\mathbf{v})$, where $n$ is as in the hypothesis $(n=1$ for $a<0)$. Note that $n=\left\lfloor\frac{\pi}{\theta}\right\rfloor$.

Now, note that a periodic point of $S_{a b}$ satisfying the constraints must be a real eigenvector of the matrix $M=\left[\begin{array}{cc}a & -1 \\ 1 & 0\end{array}\right]^{n}\left[\begin{array}{cc}b & -1 \\ 1 & 0\end{array}\right]$. Since this matrix has determinant 1 , a necessary condition is that its trace be at least 2 (if the trace were negative, the sign condition would necessarily be violated). This gives a polynomial inequality in $a$ and $b$; in terms of $\theta$, this is

$$
\sin ((n+1) \theta) b \geq 2 \sin (n \theta)+2 \sin (\theta) .
$$

Note that the choice of $n$ forces $\sin ((n+1) \theta) \leq 0$, so we have:

$$
b \leq 2 \frac{\sin (n \theta)+\sin (\theta)}{\sin ((n+1) \theta)} .
$$

Now, define $c \geq 0$ by $c \sin (\theta)=b \sin ((n+1) \theta)-2 \sin (n \theta)-2 \sin (\theta)$. Then the eigenvector of $M$ of eigenvalue $\geq 1$ is:

$$
\mathbf{v}=\left(\frac{\sin ((n+1) \theta)}{\sin (\theta)}, \frac{1}{2}(c-\sqrt{c(c+4)})+1+\frac{\sin (n \theta)}{\sin (\theta)}\right) .
$$

It remains only to verify that $\mathbf{v}$ satisfies the sign conditions. Now, $v_{x} \leq 0$ by inspection, so we need consider only $x_{i}, 1 \leq i \leq n$. By explicitly performing the appropriate matrix multiplication, we get:

$$
x_{m}=\frac{\sin (m \theta)}{\sin (\theta)} k+\frac{\sin ((n-m+1) \theta)}{\sin (\theta)},
$$

for $1 \leq m \leq n$, where $k=1+\frac{1}{2}(c+\sqrt{c(c+4)})$. This is clearly positive for all $1 \leq m \leq n$, so the result is shown.

The remaining upper bound case for $a<-2$ is handled by rewriting the proof above explicitly in terms of $a$ for the $n=1$ case; we omit the details.

Proof of Theorem 2.1, By Theorem B.2(i) the rotation number $r\left(S_{a b}\right)$ is nonincreasing in $b$, and by Theorem 3.2 it has rotation number attaining the endpoint values of the given intervals, in cases (i)-(iii). It remains only to show that for each $a, r\left(S_{a b}\right)$ is continuous in $b$, for it must then fill out the whole interval.

We first show that for varying $b, r\left(S_{a b}\right)$ assumes every rational value in the given interval. The proof of Theorem 3.2 shows that $S_{a b}(0)$ moves clockwise continuously in $a$ as $a$ increases. It follows that $S_{a b}^{(n)}(0)$ has the same behavior for each $n$. If $r=\frac{p}{q}$ is interior to the interval, 
then examining $S_{a b}^{(q)}(0)$ as $b$ increases, its continuous variation in $b$ requires it to pass through a fixed point $S_{a b}^{(q)}(0)=0$ such that $\theta=0$ is a periodic point of rotation number $\frac{p}{q}$, because if it does not, the rotation number never increases beyond $\frac{p}{q}$, a contradiction.

Any nondecreasing function $f: \mathbb{R} \rightarrow[a, b]$ which takes every rational value in $[a, b]$ is continuous. The nondecreasing property guarantees that the left limit

$$
\lim _{x \searrow x_{0}^{+}} f(x)=f^{+}\left(x_{0}\right)
$$

and right limit

$$
\lim _{x \nearrow x_{0}^{-}} f(x)=f^{-}\left(x_{0}\right)
$$

both exist, with $f^{-}\left(x_{0}\right) \leq f^{+}\left(x_{0}\right)$. If $f^{-}\left(x_{0}\right)<f^{+}\left(x_{0}\right)$, then since $f\left(x_{0}\right)$ can take only one rational value in the interval $\left(f^{-}\left(x_{0}\right), f^{+}\left(x_{0}\right)\right)$, some rational value is omitted from the range of $f(x)$, a contradiction. Thus $f^{-}\left(x_{0}\right)=f^{+}\left(x_{0}\right)$ and $f(x)$ is continuous at $x_{0}$.

Proof of Theorem 2.2. Let $\mu$ be fixed. Theorem 3.2(i) shows that $r\left(S_{\mu \nu}\right)$ is nondecreasing in $\nu$, and $r\left(S_{\mu \nu}\right) \in\left[0, \frac{1}{2}\right]$ by Theorem 3.4. We have $r\left(S_{\mu \nu}\right)=0$ when $\nu \geq 2-\mu$ by Theorem 3.4(iii) since $a=\mu+\nu \geq 2$. For $\nu$ negative and large the matrix $M=\left[\begin{array}{cc}b & -1 \\ 1 & 0\end{array}\right]\left[\begin{array}{cc}a & -1 \\ 1 & 0\end{array}\right]$ has real eigenvalues with eigenvector of $\operatorname{sign}(+,-)$ hence $S_{\mu \nu}$ has periodic point of period 2 and $r\left(S_{\mu \nu}\right)=2$.

To establish continuity of $r\left(S_{\mu \nu}\right)$ in $\nu$, it suffices to show that $S_{\mu \nu}$ has periodic points with rotation number $\frac{p}{q}$ for all rational values $0<\frac{p}{q}<\frac{1}{2}$. This follows similarly to the proof of Theorem 2.1] (We will later prove a stronger result in Lemma 4.1)

In the special cases where $S_{a b}^{(n)}(0,1)=(0, \pm 1)$ or $S_{a b}^{(n)}(0,-1)=(0, \pm 1)$ we get significant information about the behavior of $T_{a b}$.

Theorem 3.4 Let $n \geq 1$ and suppose that $S_{a b}^{(n)}(0,1)=(0, \pm 1)$, or $S_{a b}^{(n)}(0,-1)=(0, \pm 1)$, Then one of the following two relations holds:

$$
\begin{gathered}
T_{a b}^{(n)}(0,1)=(0, \lambda), \\
T_{a b}^{(n)}(0,-1)=\left(0,-\lambda^{-1}\right) .
\end{gathered}
$$

where $\lambda$ is a nonzero real number.

(i) If $\lambda>0$, then both relations above hold. In addition,

$$
T_{a b}^{(n)}(-1,0)=(-\lambda, 0) \quad \text { and } \quad T_{a b}^{(n)}(1,0)=\left(\lambda^{-1}, 0\right) .
$$

The rotation number $r\left(S_{a b}\right)$ is rational.

(ii) If $\lambda<0$, then necessarily $\lambda=-1$. In the first case

$$
T_{a b}^{(n)}(0,1)=(0,-1) \quad \text { and } \quad T_{a b}^{(n)}(-1,0)=(1,0),
$$

while in the second case,

$$
T_{a b}^{(n)}(0,-1)=(0,1) \quad \text { and } \quad T_{a b}^{(n)}(1,0)=(-1,0) .
$$

The rotation number $r\left(S_{a b}\right)$ can be irrational or rational. 
Proof. We check that the involution $R(x, y)=(y, x)$ has

$$
T_{a b}^{-1}(x, y)=R^{-1} \circ T_{a b} \circ R
$$

We have $T_{a b}(x, y)=(a x-y, x)$ if $x \geq 0$, and $(b x-y, x)$ if $x<0$. This gives

$$
T_{a b}(x, y)=(z, w) \Rightarrow T_{a b}(w, z)=(y, x),
$$

where in fact $w=x$, which implies (3.10). By induction on $n \geq 1$ this yields the implication

$$
T_{a b}^{(n)}(x, y)=(z, w) \Rightarrow T_{a b}^{(n)}(w, z)=(y, x) .
$$

Now suppose the first relation (3.5) holds. This yields

$$
T_{a b}^{(n)}(-1,0)=T_{a b}^{(n+1)}(0,1)=T_{a b}(0, \lambda)=(-\lambda, 0) .
$$

Using (3.11) applied to the first relation and to this relation yields

$$
\begin{aligned}
T_{a b}^{(n)}(\lambda, 0) & =(1,0), \\
T_{a b}^{(n)}(0,-\lambda) & =(0,-1) .
\end{aligned}
$$

If $\lambda>0$ then rescaling these by a factor $\lambda^{-1}$ gives (i), e.g.

$$
T_{a b}^{(n)}(0,-1)=\lambda^{-1} T_{a b}^{(n)}(0,-\lambda)=\left(0,-\lambda^{-1}\right)
$$

If $\lambda<0$, then we obtain:

$$
(-\lambda, 0)=T_{a b}^{(n)}(-1,0)=|\lambda|^{-1} T_{a b}^{(n)}(\lambda, 0)=\left(-\lambda^{-1}, 0\right)
$$

and thus $\lambda=\lambda^{-1}=-1$. By hypothesis $T_{a b}^{(n)}(0,1)=(0,-1)$ and applying $T_{a b}$ to both sides gives the other relation.

Now suppose the second relation (3.6) holds. This yields

$$
T_{a b}^{(n)}(1,0)=T_{a b}^{(n+1)}(0,-1)=T_{a b}\left(0,-\lambda^{-1}\right)=\left(\lambda^{-1}, 0\right) .
$$

Using (3.11) applied to these relations yields

$$
\begin{aligned}
T_{a b}^{(n)}\left(-\lambda^{-1}, 0\right) & =(0,-1) \\
T_{a b}^{(n)}\left(0, \lambda^{-1}\right) & =(0,1) .
\end{aligned}
$$

If $\lambda>0$ rescaling these equalities by $\lambda$ gives (i). The case $\lambda<0$ giving the second case in (ii). is done similarly to the first case.

If $\lambda>0$ in the first or second relation then $S_{a b}^{(n)}(0,1)=(0,1)\left(\right.$ resp. $\left.S_{a b}^{(n)}(0,-1)=(0,-1)\right)$ so the rotation number $r\left(S_{a b}\right)$ is rational.

If $\lambda<0$ in these relations there is no constraint on the rationality of the rotation number $r\left(S_{a b}\right)$. In part II we give a case (ii) example having with irrational rotation number (15. Example 4.1]), and also a case (ii) example with rational rotation number ([15, Example 4.3]).

There are several special cases in which one can give explicit formulae for the rotation number $r\left(S_{a b}\right)$. These are of interest in part because we have no formulae for generic $a$ and $b$ and in part because they also provide several examples of parameter values for which $T_{a b}$ is periodic. These results all appear in Beardon, Bullett and Rippon [2].

Example 3.1 If $a=b=2 \cos (\theta)$, with $0<\theta<\pi$, then $r\left(S_{a b}\right)=\frac{\theta}{2 \pi}$. 
Proof. Note that $T_{a b}$ is a linear map, since $a=b$. If we conjugate $T_{a b}$ by the matrix $M=\left[\begin{array}{cc}1 & 0 \\ \cos (\theta) & \sin (\theta)\end{array}\right]$, then we get the matrix $\left[\begin{array}{cc}\cos (\theta) & -\sin (\theta) \\ \sin (\theta) & \cos (\theta)\end{array}\right]$, which is rotation by $\theta$. Since $M$ is orientation-preserving for $0<\theta<\pi$, conjugation by $M$ preserves the rotation number of $S_{a b}$; rotation by $\theta$ has rotation number $\frac{\theta}{2 \pi}$, thus the formula given. Note that when $\frac{\theta}{2 \pi}$ is rational, $T_{0 \nu}$ is periodic, and the period is computed from the rotation number.

Example 3.2 If $a=2 \cos \left(\frac{\pi}{n}\right)$, for $n \geq 2$, and $b=2 \cos (\theta)$ with $0<\theta<\pi$, then $r\left(S_{a b}\right)=\frac{\theta}{\pi+n \theta}$. When $\frac{\theta}{2 \pi}=\frac{p}{q}$ is rational, then $T_{a b}$ is periodic with period $2 n p+q$, for $q$ odd, and half this if $q$ is even.

Proof. Here, we have a slightly more complicated situation, in that the map $T_{a b}$ is not linear. Again, we conjugate by $M=\left[\begin{array}{cc}1 & 0 \\ \cos (\theta) & \sin (\theta)\end{array}\right]$, to get

$$
T^{\prime}(\mathbf{v})= \begin{cases}\mathbf{v}\left[\begin{array}{ll}
\cos (\theta) & -\sin (\theta) \\
\sin (\theta) & \cos (\theta)
\end{array}\right], & \text { if }(\cos (\theta), \sin (\theta)) \cdot \mathbf{v} \leq 0 \\
\mathbf{v} M\left[\begin{array}{rr}
2 \cos \left(\frac{\pi}{n}\right) & -1 \\
1 & 0
\end{array}\right] M^{-1}, & \text { if }(\cos (\theta), \sin (\theta)) \cdot \mathbf{v} \leq 0\end{cases}
$$

The important thing to note is that the second case will always occur exactly $n$ times in a row, and that the $n$th power of that matrix is -1 . If, in formula (3.1), we consider only the subsequence of iterates not in the middle of such a block, we get the following:

$$
r\left(S_{a b}\right)=\lim _{i \rightarrow \infty} \frac{i \frac{\theta}{2 \pi}+\frac{1}{2}\left\lfloor i \frac{\theta}{\pi}\right\rfloor}{i+\left\lfloor i \frac{\theta}{\pi}\right\rfloor n} .
$$

In the limit, we can ignore the floors, to get $\frac{\theta}{\pi+n \theta}$, as claimed. Here, again, when $\frac{\theta}{2 \pi}$ is rational, $T_{a b}$ is periodic.

As a special case of Example 3.2 in $(\mu, \nu)$ parameters, taking $\nu=0, \mu=2 \cos \frac{\pi}{n}$, for $n \geq 3$, yields a $T_{\mu \nu}$ which is periodic with period $n^{2}$. This corresponds to the parameters $a=2 \cos \frac{\pi}{n}$, $b=2 \cos \frac{(n-1) \pi}{n}$ in Example 3.2 .

Example 3.3 For each integer $n \geq 2$, if both $a, b \leq 0$, and $a b=4 \cos ^{2}\left(\frac{\pi}{2 n}\right)$, then $r\left(S_{a b}\right)=\frac{2 n-1}{4 n}$, and $T_{a b}$ is periodic.

Proof. For this case, the simplest approach is to explicitly calculate $T_{a b}^{(m)}(\mathbf{v})$, for $1 \leq m \leq 4 n$, and plug that information into equation (3.1). It is most convenient to take $\mathbf{v}_{x}, \mathbf{v}_{y}<0$. We can now calculate:

$$
\begin{aligned}
x_{2 i} & =\frac{\sin \left(\frac{(i+1) \pi}{n}\right)+\sin \left(\frac{i \pi}{n}\right)}{\sin \left(\frac{\pi}{n}\right)} \mathbf{v}_{x}-\frac{\sin \left(\frac{i \pi}{n}\right)}{\sin \left(\frac{\pi}{n}\right)} a \mathbf{v}_{y} \leq 0 \\
x_{2 i+1} & =\frac{\sin \left(\frac{(i+2) \pi}{n}\right)+2 \sin \left(\frac{(i+1) \pi}{n}\right)+\sin \left(\frac{i \pi}{n}\right)}{\sin \left(\frac{\pi}{n}\right)} \frac{\mathbf{v}_{x}}{a}-\frac{\sin \left(\frac{(i+1) \pi}{n}\right)+\sin \left(\frac{i \pi}{n}\right)}{\sin \left(\frac{\pi}{n}\right)} \mathbf{v}_{y} \geq 0
\end{aligned}
$$

for $0 \leq i \leq(n-1)$. One can now calculate that $T_{a b}^{(2 n)}(\mathbf{v})=-\mathbf{v}$; since, by symmetry, we could as easily have started with $\mathbf{v}_{x}, \mathbf{v}_{y}>0$, we can conclude that $T_{a b}^{(4 n)}=1$. By inspecting the sequence 
of quadrants we pass through in a period, we conclude that in $4 n$ steps, we wrap around the origin $2 n-1$ times; $r\left(S_{a b}\right)$ is thus $\frac{2 n-1}{4 n}$.

Example 3.3 gives a countable collection of one-parameter families, on which $T_{a b}$ is a periodic map ; the question of whether other any other continuous families of periodic parameter values exist is still open. Example 4.1 in part II gives a countable number of periodic maps with isolated periodic parameter values. It gives a one-parameter family with fixed symbolic dynamics, where the rotation number changes in the family. The fixing of the symbolic dynamics bt $T_{a b}^{(8)}(0,-1)=(0,1)$ yields a one-parameter family and since the rotation number varies in the family, the rational rotation number points, which give periodic maps, must have isolated parameter values.

\section{Rational Rotation Number}

We first characterize those cases when $T_{a b}$ is a periodic map.

Proof of Theorem 2.3. If $T_{a b}$ is periodic, then $(0,1)$ is a periodic point.

Suppose, conversely, that $(0,1)$ is a periodic point of $T_{a b}$ of minimal period $p$. Then so is $(1,0)$, with the same period $p$, because $T_{a b}^{-1}$ is obtained from $T_{a b}$ by interchanging $x$ and $y$ coordinates, cf. Theorem 3.4. Next $T_{a b}((0,-1))=(1,0)$ hence $(0,-1)$ is a periodic point. We use the piecewise linear nature of $T_{a b}$ to prove that $T_{a b}$ is periodic. An iterate $T_{a b}^{(n)}$ is linear on a sector $\Sigma$ as long as the $x$-axis never intersects the interior of $T_{a b}^{(j)}(\Sigma)$ for $0 \leq j<n$. Because $(0,1)$ and $(0,-1)$ are both periodic, the image of the $x$-axis under all iterates $\left\{T^{(n)}: n>0\right\}$ splits the plane into a finite number of sectors, and the image of each of these sectors under $T$ is another sector. It follows that $T_{a b}^{(p)}$ permutes these sectors and is linear on each one of them. But each sector contains two linearly independent fixed points of $T_{a b}^{(p)}$, from which it follows that $T_{a b}^{(p)}$ is the identity map on each sector. Since these sectors partition the entire plane, $T_{a b}$ is periodic with period $p$.

The parameter values giving periodic maps $T_{a b}$ can be classified by the period length $p$, and by the symbolic dynamics of the iterates, in which the symbolic dynamics describes the $x$-coordinate signs of each iterate as belonging to $\{+,-, 0\}$. There are a countable collection of such data. In each case, either one gets (one or two) isolated parameter values, or else a one-parameter family of values $(a, b)$; the family is determined by the requirement that $T_{a b}^{(p)}(0,1)=(0,1)$, cf. Theorem 3.4. Example 3.3 exhibited a countable number of oneparameter families of periodic maps. There are a countable number of parameter values that are isolated in the sense that they are the unique point in some open neighborhood in the parameter space for a periodic map with the given period and symbolic dynamics. Examples appear in part II, as described at the end of $\S 3$.

We also note that the parameter values for periodic points can be subdivided into two types.

Type (i). $(0,1)$ and $(0,-1)$ are in the same periodic orbit of $T_{a b}$, necessarily of even period.

Type (ii). $(0,1)$ and $(0,-1)$ are in separate periodic orbits of $T_{a b}$, having the same (even or odd) period. 
Examples show that both types of periodic orbit occur.

To prove Theorem 2.4 which characterizes the possible dynamics of $T_{a b}$ when the rotation number is rational, we use the $(\mu, \nu)$-parameter space. The following auxiliary lemma is a main tool in proving Theorem 2.4 its important feature is the uniqueness assertion.

Lemma 4.1 For every rational $r=\frac{p}{q}$ with $0<r<\frac{1}{2}$ and each fixed $\mu \in \mathbb{R}$, the following holds. For each $\mathbf{v}=e^{i \theta} \in S^{1}$ there is a unique parameter value $\nu \in \mathbb{R}$ such that $\mathbf{v}$ is a periodic point of $S_{\mu \nu}$ of rotation number $r$.

Proof. Let $\mu$ be fixed. We first show that for fixed $\theta$, the second iterate $S_{\mu \nu}^{(2)}(\theta)$ moves strictly clockwise as $\nu$ increases. The proof of Theorem 3.2(ii) shows that $\frac{d}{d \nu} S_{\mu \nu}^{(2)}(\theta) \leq 0$. If $x_{0}=\mathbf{v}_{x}$ and $x_{1}=\left(S_{\mu \nu}(\mathbf{v})\right)_{x}$ then for $\frac{d}{d \nu} S_{\mu \nu}^{(2)}(\mathbf{v})=0$ it is necessary that $x_{0}=x_{1}=0$. But if $x_{0}=0$, then $x_{1}=-\mathbf{v}_{y}$ which is impossible for $\mathbf{v} \in S^{1}$. By the same argument, for each $n \geq 2$, and fixed $\theta$, $S_{\mu \nu}^{(n)}(\theta)$ moves strictly clockwise as $\nu$ increases.

The families of Example 3.3 give us some $\nu_{0}$ such that $\mathbf{v}$ is a periodic point of $S_{\mu \nu_{0}}$ of rotation number $\frac{2 q-1}{4 q}$; consider, now, the behavior of $S_{\mu \nu}^{(4 q)}(\mathbf{v})$ as $\nu$ changes from $\nu_{0}$. If $\nu$ decreases from $\nu_{0}, r\left(S_{\mu \nu}\right)>\frac{2 q-1}{4 q}>r$, so we need consider only $\nu>\nu_{0}$. As we increase $\nu, \mathbf{w}_{\nu}:=S_{\mu \nu}^{(4 q)}(\mathbf{v})$ moves smoothly (and strictly) clockwise. If we view this as a function of $\nu$ to the point $\theta_{\nu}$ with $\mathbf{w}_{\nu}=e^{2 \pi i \theta_{\nu}}$, we can take the lift $\tilde{S}(\nu)=\theta_{\nu}$ of this function to $\mathbf{R}$, with the initial condition $\tilde{S}\left(\nu_{0}\right)=2 q-1$; this is continuous, strictly decreasing, and for large enough $\nu$ attains a value below 4. $\left(\tilde{S}(\nu) \geq 4\right.$ implies $r\left(S_{\mu \nu}\right) \geq \frac{1}{q}$.) Since $2 q-1>4 p \geq 4$, there is a unique $\nu$ such that $\tilde{S}(\nu)=4 p$. It is then easy to see that $\mathbf{v}$ is a periodic point of $S_{\mu \nu}$ of rotation number $r=\frac{p}{q}$.

Proof of Theorem 2.4. We use the $(\mu, \nu)$-parameter space. We fix $\mu$, and allow $\nu$ to vary; we can use the monotonicity in $\nu$ to deduce the behavior for every $\nu$ (for that rotation number) from the behavior from one special $\nu$. In particular, let $\nu_{0}$ be the unique $\nu$ such that $(0,1)$ is a periodic point of $S_{\mu \nu_{0}}$ of rotation number $r\left(S_{\mu \nu}\right)=\frac{p}{q}$. Now, if $(0,1)$ is a periodic point of $T_{\mu \nu_{0}}$, then $T_{\mu \nu_{0}}$ is periodic, by Theorem 2.3. since rational rotation number implies existence of a periodic point, the uniqueness part of Lemma 4.1 then tells us that $\nu=\nu_{0}$ is the only value of $\nu$ for which $r\left(S_{\mu \nu}\right)=\frac{p}{q}$. This situation corresponds to case (iii) of the theorem, because we will show below that in all remaining cases $S_{a b}$ has at most two periodic orbits.

Thus, in what follows we assume that $(0,1)$ is not a periodic point of $T_{\mu \nu_{0}}$, so the map is not a periodic map. Then there must be some positive real $\lambda \neq 1$ such that $T_{\mu \nu_{0}}^{(q)}(0,1)=(0, \lambda)$. We can, without loss of generality, assume that $\lambda>1$; the proof for $\lambda<1$ is analogous. For the orbit of $(0,-1)$, we also have by Theorem [3.4(i) that $T_{\mu \nu_{0}}^{(q)}(0,-1)=\left(0,-\lambda^{-1}\right)$.

Note first that if we plot the orbits of $(0,1)$ and $(0,-1)$ under $S_{\mu \nu_{0}}$, then the two orbits are alternating as we move around the circle. Also, the sector bounded by a point of one orbit and a neighboring point from the other orbit is linear under the action of $T_{\mu \nu_{0}}^{(q)}$. Now in each sector $T_{\mu \nu_{0}}^{(q)}$ has two eigenvectors, given by the endpoints of the sector, so it can have no other eigenvector in the sector, unless it is a multiple of the identity on the whole sector. But this cannot happen, for if $T_{\mu \nu_{0}}^{(q)}$ were a multiple of the identity on the sector, necessarily $\pm I$, then each endray would consists of periodic points of period $q$ or $2 q$, and the orbit of some point 
on one of these endrays contains $(0,1)$, so $(0,1)$ would be a periodic point, contradicting our assumption. We conclude that $S_{\mu \nu_{0}}$ has no other periodic orbits.

Now, for any $0 \leq n<q$, let $\mathbf{v}_{0}(\nu) \in S^{1}$ be $S_{\mu \nu}^{(-n)}(0,1)$, and let $\mathbf{v}_{1}(\nu)$ and $\mathbf{v}_{-1}(\nu)$ be the points of the form $S_{\mu \nu}^{(-i)}(0,-1), 0 \leq i<q$ immediately counterclockwise and clockwise of $\mathbf{v}_{0}(\nu)$, respectively. For every point in the interval from $\mathbf{v}_{-1}(\nu)$ to $\mathbf{v}_{1}(\nu)$, label the point as clockwise if $S_{\mu \nu}^{(q)}$ moves the point clockwise, and similarly label it as counterclockwise if $S_{\mu \nu}^{(q)}$ moves the point counterclockwise (fixed points will receive no label). Now, at $\nu=\nu_{0}$, the points between $\mathbf{v}_{-1}$ and $\mathbf{v}_{0}$ will be labelled clockwise, and the points between $\mathbf{v}_{0}$ and $\mathbf{v}_{1}$ will be labelled counterclockwise, since $\mathbf{v}_{0}$ is an attracting fixed point of $S_{\mu \nu}^{(q)}$, and $\mathbf{v}_{ \pm 1}$ are repelling fixed points of $S_{\mu \nu}^{(q)}$. As $\nu$ decreases, every point labelled counterclockwise will remain labelled counterclockwise (by the proof of Theorem [2.2); further, $\mathbf{v}_{0}$ and $\mathbf{v}_{ \pm 1}$ will be labelled counterclockwise. Now, $T_{\mu \nu}^{(q)}$ is linear on the interval from $\mathbf{v}_{0}$ to $\mathbf{v}_{1}$, so $S_{\mu \nu}^{(q)}$ can have at most two fixed points in the interval. As long as there are points labelled clockwise in the interval, $S_{\mu \nu}^{(q)}$ must have exactly two fixed points; these must move continuously toward each other; when the two fixed points coincide, we are left with one fixed point; after that point, there can be no fixed points in the interval. Thus, we can conclude that the interval from $\mathbf{v}_{-1}$ to $\mathbf{v}_{1}$ always has either two, one, or zero fixed points of $S_{\mu \nu}^{(q)}$, in the range of $\nu \leq \nu_{0}$ such that $r\left(S_{\mu \nu}\right)=\frac{p}{q}$; the proof for $\nu \geq \nu_{0}$ is analogous. Now, the proof applies for any $\mathbf{v}_{0}$; there are exactly $q$ possible choices of $\mathbf{v}_{0}$, and the intervals from $\mathbf{v}_{-1}$ to $\mathbf{v}_{1}$ around these choices of $\mathbf{v}_{0}$ exhaust $S^{1}$; therefore, we can conclude that the number of distinct periodic orbits of $S_{\mu \nu}$ must equal the number of distinct fixed points of $S_{\mu \nu}^{(q)}$ between $\mathbf{v}_{-1}$ and $\mathbf{v}_{1}$, which is at most two. There are then three cases:

Case 1: $S_{\mu \nu}$ has no periodic orbit.

Then $r\left(S_{\mu \nu}\right)$ is irrational, contradicting the hypothesis that it is rational; this case cannot occur.

Case 2: $S_{\mu \nu}$ has exactly two periodic orbits.

Let $\mathbf{w}$ be a fixed point corresponding to one of the orbits, and $\mathbf{w}^{\prime}$ a fixed point corresponding to the other orbit. Then there must be some $\lambda$ such that $T_{\mu \nu}^{(q)}(\mathbf{w})=\lambda \mathbf{w}$, and $T_{\mu \nu}^{(q)}\left(\mathbf{w}^{\prime}\right)=\lambda^{-1} \mathbf{w}^{\prime}$. For every point $\mathbf{v}, S_{\mu \nu}^{(i)}(\mathbf{v})$ comes arbitrarily close to $\mathbf{w}$ (for $i>0$ ); there is an interval around w which behaves linearly under $T_{\mu \nu}^{(q)}$, so every point eventually is transformed by the same map. $T_{\mu \nu}^{(q)}$ has an eigenvector of eigenvalue $>1$; it is easy to see that every point must diverge exponentially except for the eigenvector of eigenvalue $<1$, which converges exponentially to 0 . Similarly, in the other direction, every point diverges exponentially except for the eigenvector of eigenvalue $>1$, which converges exponentially to 0 . This is case (ii) in the theorem.

Case 3: $S_{\mu \nu}$ has exactly one periodic orbit.

If we reflect a periodic orbit of $S_{\mu \nu}$ around the line $x=y$, we must get a periodic orbit; thus, in this case, the periodic orbit must be symmetrical around $x=y$. It follows, then, that the orbit lifts to a periodic orbit of $T_{\mu \nu}\left(T_{\mu \nu}^{(q)}\right.$ and $T_{\mu \nu}^{(-q)}$ must multiply the vectors in the orbit 
by the same amount). In a sufficiently small neighborhood of a point of the orbit, $T_{\mu \nu}^{(q)}$ is linear; it has exactly one eigenvector of eigenvalue one, but has eigenvalue one with multiplicity two; it follows that every point in that neighborhood diverges linearly.

Every point on the circle has a value of $\nu$ associated to it by the above reasoning such that it is a periodic point of $S_{\mu \nu}$. By Lemma 4.1] there can be no other $\nu$ such that $S_{\mu \nu}$ has a periodic point of rotation number $\frac{p}{q}$. This establishes case (i) of the theorem.

\section{Irrational Rotation Number}

When the rotation number is irrational, the analysis of the dynamics becomes significantly more complicated. On the level of the circle maps $S_{a b}$, their smoothness properties lead to the following result.

Theorem 5.1 If the rotation number $r\left(S_{a b}\right)=r$ is irrational, then there exists a homeomorphism $h: S^{1} \rightarrow S^{1}$ such that $h \circ S_{\mu \nu} \circ h^{-1}=\Theta_{r}$, where $\Theta_{r}: S^{1} \rightarrow S^{1}$ is rotation by $r$ $(\theta \mapsto \theta+2 \pi r)$.

Proof. By Theorem $3.1 \frac{d}{d \theta} S_{a b}(\theta)$ is continuous and of bounded variation. The topological conjugacy result then follows from Denjoy's Theorem, see Nitecki [19, p. 41 and Theorem, p. 45], or Herman [10, VI.4].

This effectively characterizes the behavior of $S_{a b}$, but it still leaves several possibilities for the dynamical behavior of $T_{a b}$. In particular, it does not rule out the possibility that $T_{a b}$ has a weakly localized orbit (one that converges to 0 as $n \rightarrow \pm \infty$ ) or of a divergent orbit (one that becomes unbounded as $n \rightarrow \pm \infty$ ). We do not know if either of these possibilities occurs.

One major problem is that of establishing conditions under which $T_{a b}$ has an invariant circle. When this holds, scale invariance implies there is an invariant circle through every point (except the origin), and the dynamics is bounded. Herman [10. VIII.2.4] shows that this occurs if and only if $T_{a b}$ is topologically conjugate to a rotation of the plane, and that this holds if and only if the map $S_{a b}$ is $C^{1}$-conjugate to a rotation of the circle. Herman conjectures that there do exist maps $T_{a b}$ with $S_{a b}$ having irrational rotation such that $T_{a b}$ is not topologically conjugate to a rotation of the plane.

We give the following condition for the existence of an invariant circle.

Theorem 5.2 Let $T: \mathbb{R}^{2} \rightarrow \mathbb{R}^{2}$ be any homeomorphism which is scale-invariant; that is $T(\lambda \mathbf{v})=\lambda T(\mathbf{v})$ for all $\lambda \geq 0$, all $\mathbf{v} \in \mathbb{R}^{2}$. Let $S(\mathbf{v})=\frac{T(\mathbf{v})}{\|T(\mathbf{v})\|}$ be its associated circle map, and suppose that $S$ is topologically conjugate to an irrational rotation. If $T$ has a forward orbit $\mathcal{O}^{+}\left(\mathbf{v}_{0}\right)=\left\{\mathbf{v}_{n}: n \geq 0\right\}$ such that

$$
\frac{1}{C} \leq\left\|\mathbf{v}_{n}\right\| \leq C, \quad \text { all } \quad n \geq 0
$$

for some positive constant $C>1$, then $T$ has an invariant circle, and all orbits are bounded. 
Proof. For each $n \geq 0$, let $L_{n}$ be the line segment from 0 to $T^{(n)}(\mathbf{v})$, and let

$$
I_{m}=\bigcup_{n \geq m} L_{n}, \quad \text { and } \quad I^{\prime}=\bigcap_{m \geq 0} \overline{I_{m}}
$$

Now, $T\left(I_{m}\right)=I_{m+1}$, so $T\left(\overline{I_{m}}\right)=\overline{I_{m+1}}$, and $\overline{I_{m}}$ is a decreasing sequence of sets. Therefore, we can conclude that $I^{\prime}$ is an invariant set of $T$. Furthermore, $\overline{I_{m}}$ contains a ball of radius $1 / C$ around 0 for each $m$, since $S^{(n)}(\mathbf{v})$ is dense in $S^{1}$, so $I^{\prime}$ contains a ball of radius $1 / C$.

Now, consider the set $B:=\partial I^{\prime}$, the boundary of $I^{\prime} . B$ is also an invariant set of $T$ which does not contain 0 , and it contains at least one point in every direction from 0 . If we can show that $B$ is homeomorphic to $S^{1}$, we will be done. It suffices to show that $B$ is connected, and contains at most one point in every direction from 0 ; projection onto the unit circle then provides a homeomorphism to $S^{1}$. Connectedness of $B$ follows from the fact that $I^{\prime}$ is closed, bounded, and star-convex, so we have left only to show that every ray from the origin hits $B$ in exactly one point.

Suppose that $\mathbf{v}_{1}, \mathbf{v}_{2} \in B$, with $\mathbf{v}_{2}=k \mathbf{v}_{1}, k>1$. Then, for all $n \geq 0$,

$$
\frac{k}{C} \leq\left|T^{(n)}\left(\mathbf{v}_{2}\right)\right| \leq C,
$$

and $\left|T^{(n)}\left(\mathbf{v}_{2}\right)\right|=k\left|T^{(n)}\left(\mathbf{v}_{1}\right)\right|$. Now, $B$ contains the line segment from $\mathbf{v}_{1}$ to $\mathbf{v}_{2}$, and thus contains the line segment from $T^{(n)}\left(\mathbf{v}_{1}\right)$ to $T^{(n)}\left(\mathbf{v}_{2}\right)$ for all $n \geq 0$. These line segments have length bounded from below, all lie within the closed ball of radius $C$ around 0 , and lie on a dense set of rays. It thus follows that $\bar{B}$ has non-empty interior. But, $B$ is a boundary, so this is impossible. Therefore, every ray must hit $B$ in at most one point, so $B$ is an invariant circle.

Theorem 5.2 applies to $T_{a b}$, and gives a criterion when it has invariant circles. We conclude by establishing the reflection symmetry of invariant circles about the line $x=y$ (when they exist); this symmetry is manifest in plots of invariant circles given in part II.

Theorem 5.3 If the map $T_{a b}$ has associated irrational rotation number $r\left(S_{a b}\right)$, they any invariant circle of $T_{a b}$ is symmetric under the involution $R(x, y)=(y, x)$.

Proof. Let $\mathcal{C}$ denote an invariant circle. The conjugacy (2.5) by $R$ shows that the set $R(\mathcal{C})=$ $R^{-1}(\mathcal{C})$ is invariant under $T_{a b}^{-1}=R^{-1} \circ T_{a b} \circ R$ and hence under $T_{a b}$, so that it too is an invariant circle of $T_{a b}$. The invariant circle $\mathcal{C}$ intersects the line $x=y$, and points on this line are left fixed by $R$, so that $\mathcal{C}$ and $R(\mathcal{C})$ have at least one common point. For irrational rotation number, any two invariant circles having a common point must be identical, because they have a dense orbit in common; thus $\mathcal{C}=R(\mathcal{C})$.

\section{References}

[1] A. Avila and S. Jitomirskaya, The ten martini problem, eprint: arXiv:math.DS/0503363

[2] A. F. Beardon, S. R. Bullett and P. J. Rippon, Periodic orbits of difference equations, Proc. Roy. Soc. Edinburgh, Ser. A (Math.) 125A (1995), 657-674. 
[3] J. Bellissard, Gap labelling theorems for Schrödinger operators, in: From Number Theory to Physics, (M. Waldschmidt, P. Moussa, J.-M. Luck, C. Itzykson, Eds.), Springer-Verlag: New York 1992, pp. 538-630.

[4] J. Bellissard, Le papillon de Hofstadter (d'apres B. Helffer et J. Sjöstrand), Seminar Bourbaki, Vol. 1991/92, Astérisque No. 206 (1992), Exp. No. 745.pp. 7-39.

[5] P. Bougerol and J. Lacroix, Products of Random Matrices with Applications to Schrödinger Operators, Birkhäuser: Boston, 1985. (see p. 187ff)

[6] M. Brown, Problem 6349, Amer. Math. Monthly 90 (1983), 569. [Solution, ibid 92 (1985), 218-219.]

[7] P. Collet and Y. Levy, Ergodic properties of the Lozi mappings, Comm. Math. Phys. 93 (1984), 461-481.

[8] F. Delyon and D. Petritis, Absences of localization in a class of Schrödinger operators with quasiperiodic potential, Comm. Math. Phys. 103 (1986), 441-444.

[9] C. Froeschlè, Étude numérique de transformations ponctuelles planes conservant les aires, C. R. Acad. Sci. Paris 266 (1968), 846-848.

[10] M. Herman, Sur la conjugasion differentiable des difféomorphismes du cercle. Publ. Math. IHES 49 (1979), 5-234.

[11] M. Herman, Sur les Courbes Invariantes par les Difféomorphismes de l'Anneau, Vol. 2 Astérisque 144, Soc. Math. de France: Paris 1986.

[12] D. Hofstadter, Energy levels and wave functions of Bloch electrons in a rational or irrational magnetic field, Phys. Rev. B 14 (1976), 2239-2249.

[13] S. Jitomirskaya, Metal-insulator transition for the almost Mathieu operator, Ann. Math. 150 (1999), 1159-1175.

[14] S. Kotani, Jacobi matrices with random potential taking finitely many values, Reviews in Math. Phys. 1 (1990), 129-133.

[15] J. C. Lagarias and E. Rains, Dynamics of a family of piecewise-linear area-preserving plane maps II. Invariant circles, eprint: arXiv:math.DS/0303007

[16] J. C. Lagarias and E. Rains, Dynamics of a family of piecewise-linear area-preserving plane maps III. Cantor set spectra, eprint: arXiv:math.DS/0505103

[17] R. Lozi, Strange attractors: a class of mappings of $\mathbb{R}^{2}$ which leaves some Cantor sets invariant, in: Intrinsic stochasticity in plasmas (Internat. Workshop, Inst. Etudes Sci. Cargèse, Cargèse 1979, Ecole Polytech., Palaiseau, 1979, pp. 373-381.

[18] M. Misiurewicz, Strange attractors for the Lozi mappings, in: Nonlinear dynamics, New York 1979, Ann. New York Acad. Sci. 357, New York Acad. Sci. 1980, pp. 348-358. 
[19] Z. Nitecki, Differentiable Dynamics: An Introduction to the Orbit Structure of Diffeomorphisms, MIT Press: Cambridge, MA 1971.

[20] L. Pastur and A. Figotin, Spectra of random and almost-periodic operators, Grund. Math. Wiss. 297, Springer-Verlag: Berlin 1992.

[21] J. Puig, Cantor spectrum for the almost Mathieu operator, Comm. Math. Phys. 244 (2004), 297-309.

[22] J. Sjóstrand, Microlocal analysis for the periodic magnetic Schrödinger equation and related questions, pp. 237-332 in: Microlocal analysis and applications (Motecatini Terme, 1989), Lecture Notes in Math. 1495, Springer-Verlag, Berlin 1991.

[23] T. Spencer, Random and Quasiperiodic Schrödinger Operators, Proc. Int. Congress Math., Vol II (Berkeley 1986), pp. 1312-1318.

[24] B. Sutherland and M. Kohmoto, Resistance of a one-dimensional quasicrystal: Power-law growth, Phys. Rev. B 36 (1987), 5877-5886.

[25] A. Sütö, Schrödinger difference equation with deterministic ergodic potentials, in: Beyond Quasicrystals (Les Houches 1994), Springer-Verlag: New York 1995, pp. 481-549.

[26] Yi Wan and C. M. Soukoulis, One-dimensional nonlinear Schrödinger equation: a nonlinear dynamics approach, Phys. Rev. A 41 (1990), 800-809. 